\title{
Morphological Evaluation of Zygomatic root of Sapajus Apella Primates
}

\author{
Maria Claudia Harada Ferreira*, Alexandre Rodrigues Freire, Rafael Araujo, Paulo Roberto Botacin, Felippe \\ Bevilacqua Prado, Ana Cláudia Rossi.
}

\begin{abstract}
The objective of this study was to evaluate the position of the maxillary premolars and molars in relation to the zygomatic root of Sapajus apella monkeys. The results suggested that there are differences in position between the premolars and molars in relation to the long axis of the zygomatic root of Sapajus apella monkeys. The third premolar is the one closest to the long axis of the zygomatic root.
\end{abstract}

Key words: anatomy, zygomatic root, biomechanics craniofacial.

\section{Introduction}

The region of the zygomatic pillar in primates, specifically in monkeys of the Sapajus genus, is called a zygomatic root and is located between the mesial alveolar bone of the maxillary first molar and the distal alveolar bone of the third premolar (Strait et al. 2008). Rak (1983) focuses on the hypothesis of three important variables for craniofacial morphology: the location of the occlusal bite force, the location of the zygomatic root, and the structural rigidity. The interpretation in which the stresses and deformations were produced by the premolar load are different from those produced by the molar load and consequently induce a craniofacial morphological adaptation (Prado et al., 2016).

The aim of this study was to evaluate the position of the maxillary premolars and molars in relation to the zygomatic root of Sapajus apella monkeys.

\section{Results and Discussion}

Twelve skulls of adult Capuchin Monkeys (Specie Sapajus apella), were used in this study. A digital caliper was used for the measurements. Measurements were made to describe the distance of each premolar and molar with the long axis of the zygomatic root on both sides. Shapiro-Wilk test was used to determine if the measurements were conformed to a normal distribution. Comparisons of side were analyzed with Student $t$ test. Tukey's post-hoc test was used to verify the difference between all relationships. All calculations were performed on $R$ CRAN Source software. In all cases, the significance level was set at $p<0.05$. The Shapiro-Wilk test showed that all groups presented a parametric distribution of the data. There was no statistically significant difference only between the relation of the third premolar and first molar groups on the right side. All other relationships showed a difference between measurements on the same side. When comparing the right side to the left side (paired Student's t-test), a statistically significant difference was found only for the second premolar group and the third molar group. Measurements of the third premolar are significantly smaller in relation to the first molar, on both sides. The results suggest that the zygomatic root in the adult dentate Sapajus apella monkey skull exhibit differences in relation to the premolars and molars axis.

\section{Conclusions}

In conclusion, the third premolar is the one closest to the long axis of the zygomatic root.

\section{Acknowledgement}

CNPq/PIBIC.

\footnotetext{
${ }^{1}$ Prado, F.B.; Freire, A.R.; Rossi, A.C.; Ledogar, J.A.; Smith, A.L.; Dechow, P.C.; Strait, D.S.; Voigt, T; Ross, C.F. Review of In Vivo Bone Strain Studies and Finite Element Models of the Zygomatic Complex in Humans and Nonhuman Primates: Implications for Clinical Research and Practice. Anat Rec (Hoboken). 2016, 299, 1753-1778.

2 Rak, Y. The Australopithecine Face. 1st ed. New York: Academic Press. 1983

${ }^{3}$ Strait, D.S.; Wright, B.W.; Richmond, B.G.; Ross, C.F.; Dechow, P.C.; Spencer, M.A.; Wang, Q. Craniofacial Strain Patterns During Premolar Loading: Implications for Human Evolution. Chapter 9. In: Vinyard C et al. (eds.), Primate Craniofacial Function and Biology, Springer Science + Business Media, LLC. 2008.
} 